\title{
Rare Adrenal Gland Emergencies: A Case Series of Giant Myelolipoma Presenting With Massive Hemorrhage and Abscess
}

\author{
Santosh $\operatorname{Kumar}^{1,}$; Kumar Jayant $^{1}$; Seema Prasad ${ }^{1}$; Swati Agrawal ${ }^{2}$; Kalpesh Mahesh Parma \\ ${ }^{1}$; Rajesh Roat ${ }^{1}$; Kushal Kumar ${ }^{1}$ \\ ${ }^{1}$ Department of Urology, Post Graduate Institute of Medical Education and Research, Chandigarh, India \\ ${ }^{2}$ Department of Obstetrics and Gynaecology, Post Graduate Institute of Medical Education and Research, Chandigarh, India \\ ${ }^{*}$ Corresponding author: Santosh Kumar, Department of Urology, Post Graduate Institute of Medical Education and Research, Chandigarh, India. Tel: +91-941737406, Fax: +91- \\ 1722744401, E-mail: santoshsp1967jaimatadi@yahoo.co.in
}

Received: August 9, 2014; Accepted: September 23, 2014

\begin{abstract}
Introduction: Adrenal Myelolipoma is a rare benign neoplasm, which contains mature adipose tissue and variable amounts of haematopoietic elements. Most lesions are small and asymptomatic, discovered incidentally during autopsy or imaging studies performed for other reasons.

Case Presentation: Here we reported a series of two cases of giant myelolipomas of the adrenal gland; first one the largest tumor reported so far presented with massive hemorrhage and the second case introduced with its rare unreported presentation of adrenal myelolipomas i.e. a large abscess.

Discussion: Adrenal myelolipoma is a rare and asymptomatic tumor usually discovered incidentally in less than $1 \%$ of population on autopsy or imaging performed for other reasons. There is an increasing incidence of large adrenal myelolipoma $(>10 \mathrm{~cm})$ presenting with life threatening and recurrent retroperitoneal hemorrhage along with other complications as abscess. To avoid such a life-threatening situation, authors recommend close monitoring and consideration of urgent surgical intervention for tumors larger than $4 \mathrm{~cm}$ at presentation or increase in size or change in appearance during follow-up.
\end{abstract}

Keywords:Myelolipoma; Hemorrhage; Abscess

\section{Introduction}

Myelolipoma was initially described by Gierke in 1905, and the term "myelolipomatoses" was coined by Oberling in 1929 (1). Adrenal myelolipoma is a very rare benign tumor. It is usually small, asymptomatic and non-functional in nature. Virtually, all adrenal myelolipomas originate from the adrenal cortex. Its overall incidence is $0.05-0.2 \%$, which accounts for about $2.5-5 \%$ of adrenal incidentalomas with both genders equally affected. They are usually unilateral and rarely arise from extra adrenal sites like retroperitoneum, thorax and pelvis (2). On histology, they have variable amounts of adipocytes with interspersed haematopoietic elements. The tumor tissue myeloid to erythroid ratio of 3 to $4: 1$ is similar to that of bone marrow, but the number of megakaryocytes may be increased. Here we reported a series of two cases of rare surgical emergencies of adrenal gland tumors. The first case was a giant adrenal myelolipoma with severe anemia secondary to massive retroperitoneal hemorrhage and the second one a giant myelolipoma presented with sepsis secondary to a large abscess.

\section{Case Presentation}

\subsection{Case 1}

A 40-year-old male patient presented to the emergency department of our institute with sudden onset of dyspnea, dizziness and pain in upper abdomen. His pulse rate was 130/minute, blood pressure of $90 / 48 \mathrm{mmHg}$, respiratory rate of $30 /$ minute and temperature of $98.60^{\circ} \mathrm{F}$. He was promptly resuscitated and blood was sent for cross-match and routine investigations. On evaluation, he was pale with a huge lump palpable in his abdomen. Blood investigations revealed hemoglobin of $5 \mathrm{~g} / \mathrm{dL}$, a total leukocyte count of $10000 / \mathrm{mm}^{3}$, a platelet count of 100 $\mathrm{x} 10^{3} / \mathrm{mm}^{3}$ and normal results of renal function tests. He received three units of packed RBC. Ultrasonography of abdomen showed a large hyperechoic suprarenal mass of $30 \times 20 \mathrm{~cm}$, which displaced the right kidney downwards. Later a computed tomography (CT) scan was performed which revealed a $35 \times 19 \mathrm{~cm}$ suprarenal mass pushing the kidney downwards. The mass was hypodense, nonenhancing with an attenuation value suggestive of fat i.e., $-19 \mathrm{HU}$ in multiple areas with intralesional hemor- 
rhage (Figure 1). Plasma biochemistry analysis showed free cortisol of $10.3 \mathrm{ug} / \mathrm{mL}$, metanephrines of $20 \mathrm{pg} / \mathrm{mL}$ and normetanephrines of $46.8 \mathrm{pg} / \mathrm{mL}$, which were within the normal limits. Later with improvement of patient's general condition, he was scheduled for laparotomy and right adrenalectomy was performed. The resected specimen was $38 \times 20 \times 16 \mathrm{~cm}$ and weighed 5200 grams with a well circumscribed tumor showing multiple areas of hemorrhage (Figures 2 and 3). The specimen was sent for histopathology, which revealed mature adipose tissue with tri-lineage hematopoietic elements without any evidence of dysplasia, which confirmed the diagnosis of adrenal myelolipoma (Figure 4). His postoperative recovery was smooth and follow-up ultrasound performed at six month showed no evidence of recurrence.

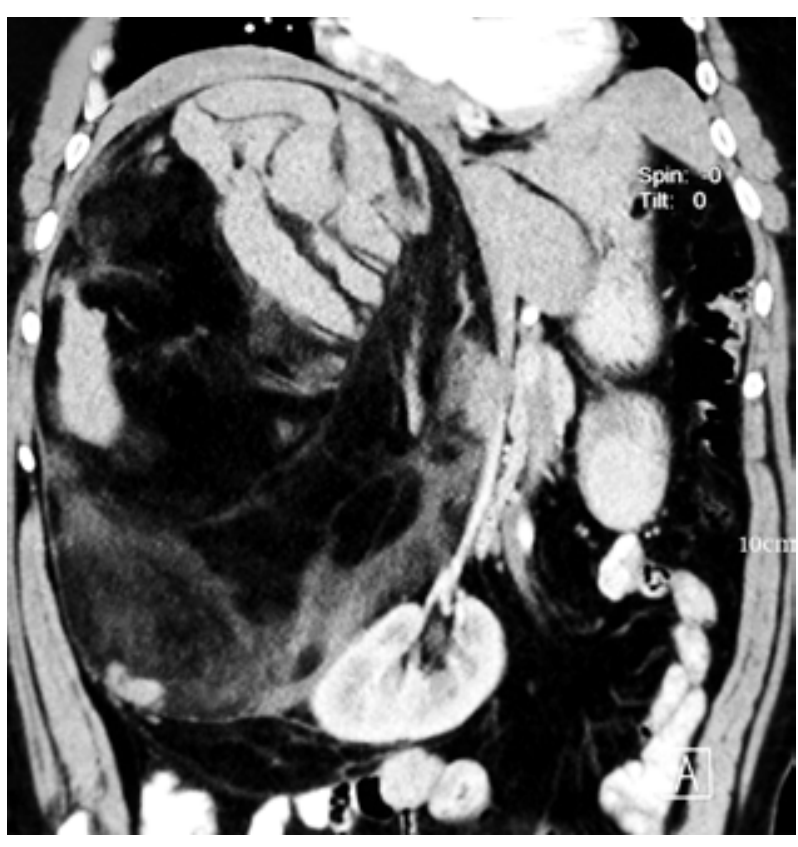

Figure 1. CECT Abdomen Showing a 35 x $19 \mathrm{~cm}$ Suprarenal Mass Hounsfield Density (HU-19)

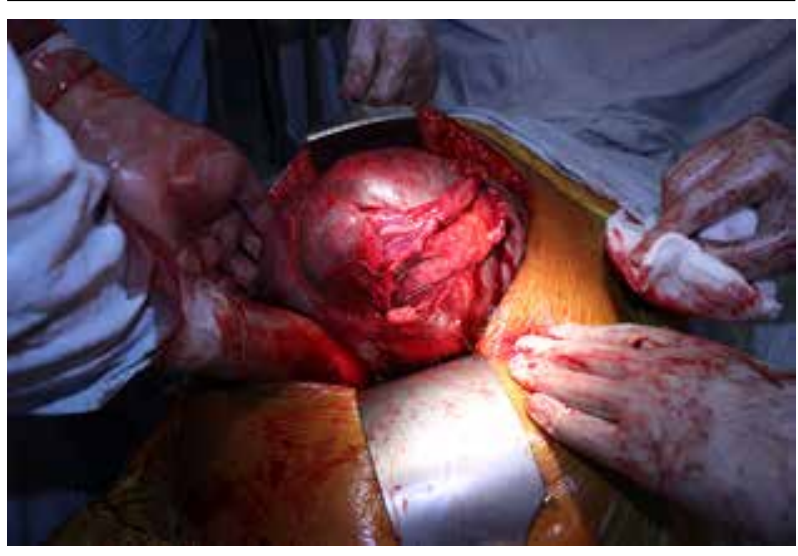

Figure 2. Intraoperative Picture Showing a Large Suprarenal Mass

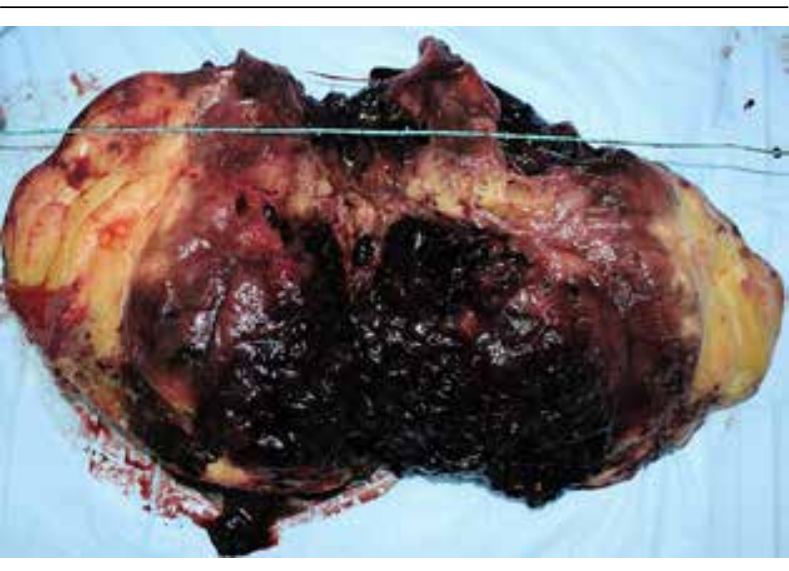

Figure 3. Resected Specimen With a Size of $38 \times 20 \times 16 \mathrm{~cm}$ and Weight of 5200 Grams With Well Circumscribed Tumor Showing Multiple Areas of Hemorrhage

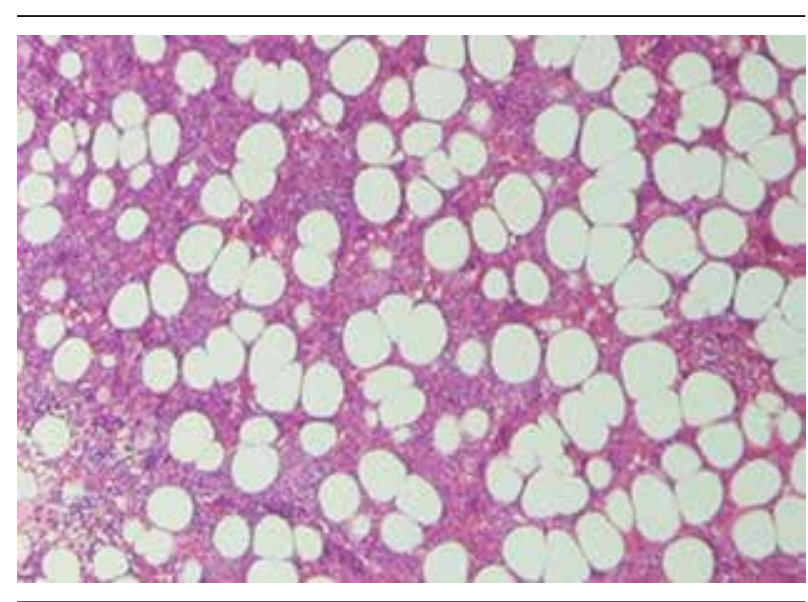

Figure 4. Histopathology Showing Adiposities Admixed With Hematopoitic Cells Suggestive of Myelolipoma of Adrenal Gland

\subsection{Case 2}

A 50-year-old male patient with diabetes presented to our emergency ward with fever, chills, rigors, pain in upper abdomen and vomiting since last five days. On examination, his pulse rate was $130 / \mathrm{min}$, blood pressure of $86 / 48 \mathrm{mmHg}$, respiratory rate of 30/minute and temperature of $103.60^{\circ} \mathrm{F}$. He was promptly resuscitated and blood sample was sent for cross-match and routine investigations. On evaluation, he was pale with a visible lump in his abdomen. Blood investigations revealed a hemoglobin of $7.8 \mathrm{~g} / \mathrm{dL}$, a total leukocyte count of $22000 / \mathrm{mm}^{3}$, and a platelet count of $108 \times 103 / \mathrm{mm}$, creatinine of $1.93 \mathrm{mg} /$ $\mathrm{dL}$ and a blood urea nitrogen (BUN) of $80 \mathrm{mg} / \mathrm{dL}$. Ultrasonography of the abdomen revealed a large hypochoic area with a size of $22 \times 17 \mathrm{~cm}$ in sub hepatic location. Later CT was performed, which revealed a $23 \times 21 \mathrm{~cm}$ right suprarenal mass with fat and fluid density within it, pushing the kidney downwards (Figure 5). Once the patient's general condition improved, he was scheduled for laparotomy and a mass was excised after draining the abscess 
(Figures 6 and 7). Postoperative recovery was uneventful. The specimen was sent for histopathological analysis, which confirmed the diagnosis of an adrenal myelolipoma. Microscopic examination revealed that the tumor included mature adipose tissue without nuclear atypia in the adipocytes, some fibroblast, focally hematopoietic cells, and areas of hemorrhage, necrosis and inflammation. The drained pus was sent for culture, which showed pseudomonas sensitive to piperacillin and tazobactam (Figure 8). Later follow-up ultrasonography performed at six month showed no recurrence of tumor.

\section{Discussion}

Adrenal myelolipoma is a rare and asymptomatic tumor usually discovered incidentally in less than $1 \%$ of population on autopsy or imaging performed for other reasons. Tumor size varies from several millimeters to more than 30 centimeters. Larger lesions (i.e. $>5 \mathrm{~cm}$ ) can

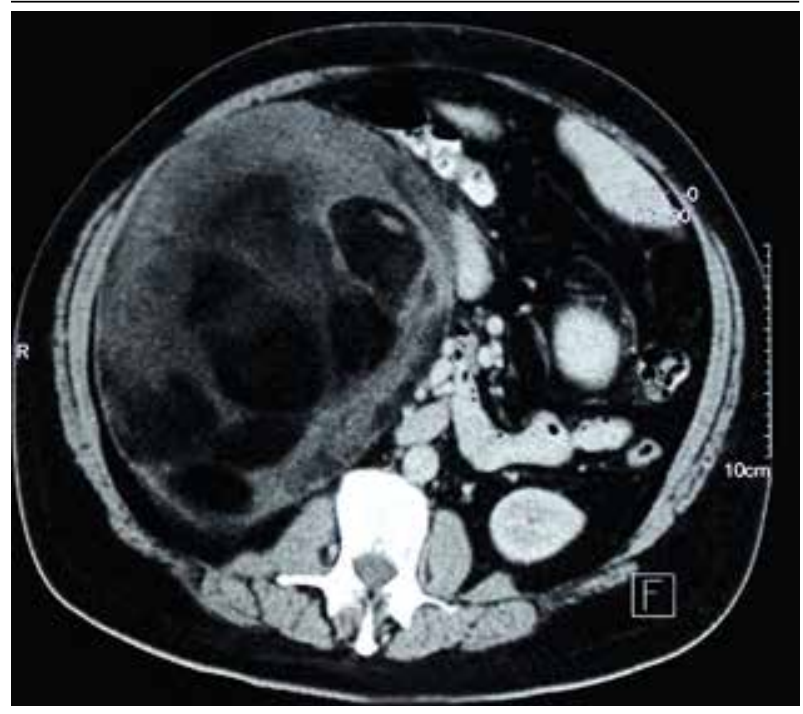

Figure 5. CECT Abdomen Showing a $23 \times 21 \mathrm{~cm}$ Suprarenal Mass With Fat and Fluid Density Within it Pushing the Kidney Downwards

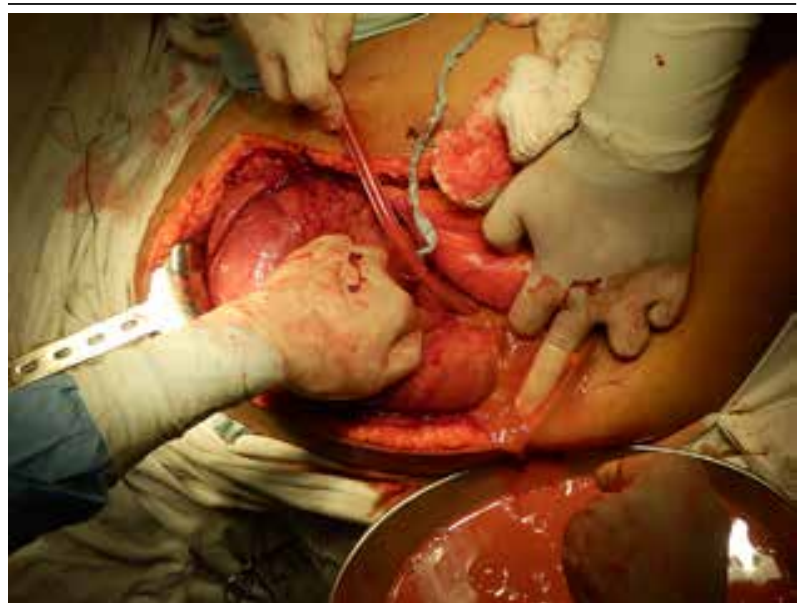

Figure 6. Intraoperative Picture Showing a Large Suprarenal Mass With Pus Filled Within it rarely present with acute retroperitoneal hemorrhage (3). The largest myelolipoma so far was reported by Boudreaux et al. which was $34 \mathrm{~cm}$ in size and weighed 5900 grams. The first case in our series had a larger tumor than that of Boudreaux et al. (4). Although the tumor itself is nonfunctioning, there is 10\% incidence of associated endocrine disorders such as Cushing's disease,

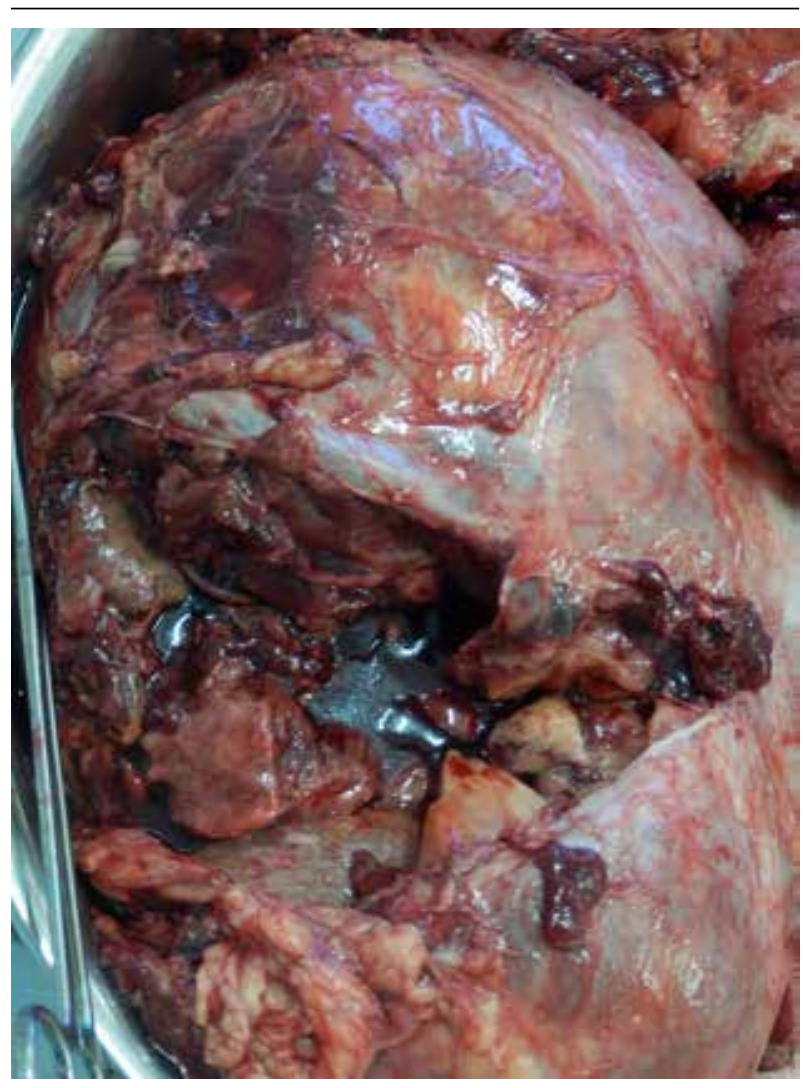

Figure 7. Resected Specimen Showing Tumor With Residual Cavity After Aspiration of Pus

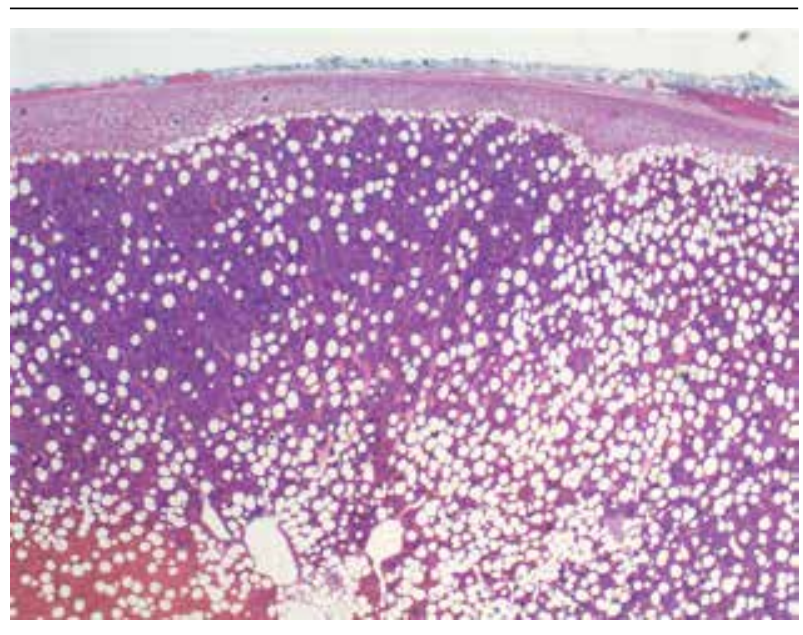

Figure 8. Histopathology Showing a Mixture of Adipose and Myeloid Elements Suggestive of Myelolipoma of the Adrenal Gland 
Conn's syndrome, diabetes and congenital adrenal hyperplasia. Besides, rare associations with sickle cell anemia and thalassemia have been reported. Although the reasons are not well known, myelolipoma in patients with thalassemia are usually giant and bilateral. Such an association might be due to excess production of erythropoietin (5). Various possible explanations exist regarding the pathogenesis of myelolipoma. The most accepted one is metaplasia of reticuloendothelial cells of blood capillaries in adrenal gland in response to various stimuli such as necrosis, inflammation, infection or stress. Whether these tumors are neoplasm or relative growth, is not clear to date. A study by Yildiz et al. showed nonrandom $X$ chromosome inactivation in hematopoietic lineage and fat cells, suggestive of a clonal origin. Similarly, another study performed by Chang et al. demonstrated a balanced translocation between $3 q$ 25 and 21q 11, which was suggestive of a possible clonality in the origin of myelolipoma. Our second case in the series presented with features of sepsis and anemia; this presentation was not reported in the literature so far. Possible explanation of such presentation may be due to infection, secondary to rupture of large tumor leading to abscess formation (6).

Diagnosis of adrenal myelolipoma is usually made by identification of fat on various imaging modalities as US, CT and MRI, which are effective to detect more than $90 \%$ of cases. Of these, CT is the most sensitive one to detect such lesions. Sonographic appearance varies with the tissue component in tumor. It appears hyperechoic if it contains fat and hypoechoic if it has predominance of myeloid cells. CT appearance also depends on tumor tissue composition. Tumor mass shows large amount of fat [attenuation $<30$ Hounsfield units (HU)] with interspersed of higher attenuation due to myeloid element. The overall attenuation values are low (-20 to -30 $\mathrm{HU}$ ) and suggestive of mixture of adipose and myeloid elements. The tumor capsule may show calcification in $20 \%$ of patients, secondary to hemorrhage. MRI characteristically demonstrates high signal intensity on $\mathrm{T} 1$ weighted images consistent of fat, which is often found in these lesions. Although, use of MRI imaging can be diagnostic (7). In cases of diagnostic dilemma, percutaneous biopsy must be considered, which can reveal mature adipocytes similar to marrow and hematopoietic cells. Histological assessment is helpful in differentiation of other tumors found in the retroperitoneal area (8). Adrenal myelolipoma are benign lesions with no recognized malignant potential. As such if imaging features are characteristic and lesion is small, recommended treatment is observation. As per the study of Meyer et al. the calculated doubling time of adrenal myelolipoma ranged from 4.6 to 95.1 months (a mean of 31.9 months), which confirmed the relatively slow growing nature of incidental myelolipoma and suggested no need for so frequent observation of myeloli- poma. However, hemorrhage and other complications occur more commonly in larger lesions (diameter $>10$ $\mathrm{cm}$ ). Therefore, only in cases of large symptomatic lesions or when hemorrhage has occurred, surgical excision is curative (9).

In our scenario, the first case was the largest adrenal myelolipoma reported so far with 38 X $20 \times 16 \mathrm{~cm}$ in size presented with severe anemia secondary to acute retroperitoneal hemorrhage. While, the second case was a large adrenal myelolipoma presented with severe sepsis secondary to abscess formation. Our both cases needed blood transfusion and urgent surgery to save their lives. We made the initial differential diagnoses of retroperitoneal liposarcoma, liver abscess, angiomyolipoma with retroperitoneal hemorrhage, since huge myelolipoma of adrenal is a very rare pathology and acute retroperitoneal hemorrhage and abscess formation is even rarer phenomenon associated with it, so we kept it as the last differential diagnosis in our list (10). Later, post resection histopathological assessment confirmed the diagnosis of an adrenal myelolipoma. There is an increasing incidence of large adrenal myelolipoma $(>10 \mathrm{~cm})$ presenting with life threatening and recurrent retroperitoneal hemorrhage along with other complications as abscess. To avoid such a life-threatening situation, authors recommend close monitoring and consideration of urgent surgical intervention for tumors larger than $4 \mathrm{~cm}$ at presentation or increase in size or change in appearance during follow-up.

\section{Acknowledgements}

The authors would like to thank patients for allowing publishing case series and using images taken during their stay in hospital.

\section{Authors' Contributions}

Santosh Kumar, Kumar Jayant and Seema Prasad developed the original concept and idea behind the manuscript. Kumar Jayant, Swati Agrawal, Kalpesh Parmar and Rajesh Roat wrote the manuscript and discussion and collected all figures. Santosh Kumar and Kumar Jayant were involved in critical revision of the manuscript and important intellectual contents.

\section{References}

1. Lawler LP, Pickhardt PJ. Giant adrenal myelolipoma presenting with spontaneous hemorrhage. CT, MR and pathology correlation. Ir Med J. 2001;94(8):231-3.

2. Kenney PJ, Wagner BJ, Rao P, Heffess CS. Myelolipoma: CT and pathologic features. Radiology. 1998;208(1):87-95.

3. Han M, Burnett AL, Fishman EK, Marshall FF. The natural history and treatment of adrenal myelolipoma. J Urol. 1997;157(4):1213-6.

4. Boudreaux D, Waisman J, Skinner DG, Low R. Giant adrenal myelolipoma and testicular interstitial cell tumor in a man with congenital 21-hydroxylase deficiency. Am J Surg Pathol. 1979;3(2):109-23.

5. Civrilli K, Damry N, Steppe R, Efira A, Mathieu J. Bilateral adrenal myelolipomas. JBR-BTR. 2008;91(3):90-1.

6. Yildiz L, Akpolat I, Erzurumlu K, Aydin O, Kandemir B. Giant adre- 


\section{Kumar Set al.}

nal myelolipoma: case report and review of the literature. Pathol Int. 2000;50(6):502-4.

7. Al Harthi B, Riaz MM, Al Khalaf AH, Al Zoum M, Al Shakweer W. Adrenal myelolipoma a rare benign tumour managed laparoscopically: Report of two cases. J Minim Access Surg. 2009;5(4):118-20.

8. Manassero F, Pomara G, Rappa F, Cuttano MG, Crisci A, Selli
C. Adrenal myelolipoma associated with adenoma. Int J Urol. 2004;11(5):326-8.

9. Meyer A, Behrend M. Presentation and therapy of myelolipoma. Int JUrol. 2005;12(3):239-43.

10. Jayant K, Agrawal S, Agarwal R, Khoiwal S. Pancreatic Ewings Sarcoma-A Dreadful Tumor. Am J Cancer Prev. 2013;1(3):24-6. 